Reprod. Nutr. Dévelop., 1980, 20 (3 B), 771-778.

\title{
Les relations parentales chez les hyménoptères sociaux
}

par P. JAISSON

Laboratoire d'Ethologie ef Sociobiologie,

Equipe de Recherche Associée au CNRS no 885

Université Poris XIII,

avenue J.-B.-Clément, 93430 Villetaneuse, France.

\section{Summary. Parental relationships in social Hymenoptera.}

The societies of Hymenoptera are family groups where several generations live together. Parental relationships are particularly developed and are usually integrated into the division of labor. Parental behaviors include mainly licking, feeding the larvae, thermoregulation and transport of the brood, aiding in pupation and hatching.

The parental relationships in social Hymenoptera are developed according to phylogenesis, in the same way as other behavioral types. These relations involve the use of specific signals, some emitted by the young and others by the adults. Some of the signals are learned during an early experience. In these insects, the progeny (or brood) constitute a basic class which is necessary to the physiological and behavioral regulations of the society.

Il existe relativement peu d'études approfondies des relations parentales chez les Hyménoptères sociaux, car ces sociétés supérieures d'Insectes ont une structure très complexe et souvent un nombre élevé de membres (quelques dizaines de milliers chez l'Abeille domestique, plusieurs millions chez les Fourmis Dorylines). Cela constitue un obstacle technique lorsqu'on essaie d'atteindre le niveau individuel pour analyser tous les aspects des relations parentales en rapport avec la division du travail et les régulations sociales. Par ailleurs, une caractéristique propre aux Hyménoptères sociaux ainsi qu'aux Termites accroît les difficultés d'interprétation par rapport aux autres Insectes ou aux Vertébrés : sauf au moment de la fondation (là où elle existe), œufs, larves et nymphes ne sont pas soignés par la mère (ou « reine ») mais par des individus généralement stériles, de sexe génétique femelle chez les Hyménoptères, les « ouvrières », qui sont en fait des sœurs aînées.

Dans le cas des Hyménoptères s'ajoute la règle de l'haplodiploïdie (sexe déterminé par le nombre de chromosomes : $n$ donne un mâle tandis que $2 n$ donne une femelle (reine ou ouvrière). Cette règle est précisément à la base de la théorie de Hamilton (1964), elle-même reprise par Wilson (1975) dans sa théorie sociobiologique de la sélection parentale. D'après ces auteurs, en effet, les "gènes sociaux » se trou- 
vent particulièrement favorisés par une structure sociale où l'altruisme que manifestenf les ouvrières est dirigé vers des génotypes qui leur sont communs à 75 p. 100 en moyenne : l'haploïdie des mâles les dispense d'une méiose, source d'hétérogénéité génétique. Ainsi, tous les spermatozoïdes stockés dans la spermathèque de la reine au terme d'une fécondation sont, théoriquement, génétiquement identiques. II en résulte que deux ouvrières entre elles seront beaucoup plus proches génétiquement qu'elles ne le sont de leur mère $(50 p$. 100 de gènes communs en moyenne au lieu des 75 p. 100 précédents). En prodiguant des soins à leur mère puis en soignant leurs futures scurs, les ouvrières favorisent donc la multiplication des $3 / 4$ de leur propre génotype. Cette théorie ne repose pas sur une démonstration directe mais suscite suffisamment d'intérêt (voire d'engouement) chez les spécialistes pour que nous la citions pour mémoire.

\section{I. - Les différents types de comportements impliqués dans les relations parentales chez les Hyménoptères sociaux.}

L'altruisme des individus soigneurs s'exprime à travers plusieurs types de comportements, d'ailleurs variables selon que les jeunes sont élevés en alvéoles (Abeilles, Bourdons et Guêpes par exemple) ou non (Fourmis).

1) Les léchages : prodigués dès le stade œuf, ils permettent de lutter contre une éventuelle déshydratation mais surtout de maintenir la propreté du couvain en éliminant déchets, défécations ou spores qui en se développant, entraîneraient le pourrissement. Ces léchages conditionnant l'intégrité du couvain peuvent se poursuivre tout au long du développemenf larvaire et parfois jusqu'au stade nymphal compris (cas des Fourmis Pseudomyrmicines, Myrmicines ou Dolichodérines, où la larve ne tisse pas de cocon protecteur avant la nymphose).

2) Le nourrissage des larves : cefte activité implique la réalisation d'une chaîne ergonomique mettant en relation individus soigneurs ef pourvoyeurs, l'alimentation des larves étant différente de celle des adultes. Ces derniers absorbent surtout des aliments d'entretient liquides, riches en glucides, tandis que les larves ingèrent des nutriments à teneur plus élevée en protéines (pollen chez les Apidés, viandes diverses chez Guêpes et Fourmis). Les individus pourvcyeurs récoltent donc, pour leur progéniture, un aliment différent de ce qu'ils consomment eux-mêmes. Dans le nid, les soigneurs assurent le relai en distribuant cette récolte à la progéniture. Les caractéristiques du nourrissage ont des conséquences importantes sur le devenir des larves à 2 n chromosomes en ouvrières ou reines. Dans certains cas la quantité d'aliments est prépondérante tandis que dans d'autres c'est la qualité de l'aliment qui joue un rôle fondamental : ainsi chez l'Abeille la sécrétion des glandes mandibulaires et hypopharyngées des ouvrières soigneuses constitue l'alimentation exclusive des larves de reines.

D'une manière générale, on admet que les ouvrières soigneuses sont plutôt des individus jeunes, tandis que les pourvoyeuses sont plutôt âgées. En affinant davantage, Fresneau et Dupuy (1980) ont démontré chez la Fourmi Neoponera apicalis que le vieillissement se traduit, sur le plan du travail social, par un éloignement progressif des 
œufs. Chez ces Fourmis, les soigneuses les plus spécifiques seraient donc celles qui s'occupent régulièrement des œufs. Ce résultat corrobore ceux de Lenoir (1977) qui utilise avec succès, chez Lasius niger, un test de transport provoqué des larves et cocons pour dépister les pourvoyeuses. Ce n'est évidemment pas l'âge en tant que tel qui se trouve responsable de l'évolution des soins mais plutôt les modifications physiologiques, essentiellement glandulaires, qu'il entraîne et avec lesquelles il est, par conséquent, corrélé (cf. par exemple les travaux classiques de Rcsch, 1930, sur l'Abeille domestique).

3) La thermorégulation ef les transports du couvain : il peut sembler curieux de classer dans une même rubrique ces deux phénomènes au demeurant fort différents. Cependant, selon le groupe d'Hyménoptère social considéré, ils remplissent la même fonction d'incubation dans des conditions optimales de température pour le couvain. Chez les Abeilles, les Bourdons et les Guêpes, où l'œuf, la larve ou la nymphe se trouve contenu dans un alvéole, la température du nid est maintenue, par différentes voies, à un niveau dépassant souvent $30^{\circ} \mathrm{C}$ (isolation thermique, chaleur des combustions organiques, ventilation, ...). Pour les Fourmis, où le ccuvain est libre de tout alvéole, la colonie peut changer de nid très facilement, le transport permettant de placer la progéniture dans les meilleures conditions d'incubation en fonction de ses caractéristiques et de l'heure de la journée. En été, par exemple, les nymphes seront ramenées tout près de la surface, voire immédiatement au contact d'une pierre bien chauffée par le soleil, puis redescendues pour la nuit où la température restera plus stable qu'en surface. En revanche, œufs et larves, nécessitant moins de chaleur, pourront rester plus en profondeur.

4) L'aide pour la nymphose ef l'éclosion : chez les Hyménoptères sociaux volants, la larve n'a généralement pas besoin de l'aide des ouvrières pour se nymphoser : elle reste enfermée dans son alvéole et peut tisser un cocon de soie plus ou moins complet contre la paroi. Pour l'éclosion imaginale, le jeune individu pourrait généralement se passer de l'aide des soigneuses; mais, parallèlement aux efforts qu'il développe pour sortir de son «carcan », des ouvrières sont attirées et mordillent l'opercule, ce qui accélère considérablement l'opération. Par ailleurs, cet opercule est souvent mouillé et ramolli par un liquide libéré lors de la mue imaginale.

Chez les Fourmis existent des sous-Familles où les larves ne tissent pas de cocon (Myrmicines, Pseudomyrmicines, Dolichodérines) et d'autres où ce travail est effectué (Myrméciines, Ponérines, Formicines, Dorylines). Dans ce dernier cas les larves s'appuient sur des matériaux-supports présents dans le milieu ou apportés spécialement par les ouvrières. Il arrive parfois qu'une partie importante des larves affeigne la nymphose sans tisser de cocon (certaines Formica). La cause en est inconnue (carence des soins, conditions écologiques, ...) mais la métamorphose n'en semble pas perturbée. Lorsqu'on élève au laboratoire certaines Fourmis Ponérines (Odontomachus ou Neoponera) dans des nids artificiels au sol lisse, l'absence de support empêche la larve de tisser son cocon, ce qui entraîne l'impossibilité de se nymphoser, donc la mort (Jaisson ef Fresneau, 1978a). D'une manière générale, il est admis que la présence du cocon de soie protège la nymphe contre les chocs, les parasites ou les moisissures mais la préserve également de la tendance au cannibalisme, répandue chez les Fourmis. Au terme de la nymphose, la sortie du cocon peut se faire sans l'intervention des 
adultes (Lasius par exemple) mais nous avons pu constater parfois que l'éclosion se faisait difficilement (Formica) ou bien était impossible (Camponotus, Odontomachus). L'aide des adultes consiste à découper transversalement le cocon puis à lécher activement le corps de la jeune, ce qui facilite le départ de l'exuvie nymphale.

5) La progéniture, réserve alimentaire de la société : il est impossible d'évoquer les relations parentales chez les Hyménoptères sociaux sans parler du flux trophique existant dans le sens larves $\rightarrow$ adultes. En effet, les larves constituent une réserve en aliments qui se trouve utilisée, notamment, lorsque des conditions écologiques momentanément défavorables rendent l'approvisionnement déficitaire. Ces échanges furent étudiés, en particulier, par Le Masne (1953) chez les Fourmis. Montagner (1967) a par ailleurs montré, chez des Guêpes du genre Vespula à la fin du cycle d'une société annuelle, que les mâles, ayant des difficultés à obtenir une régurgitation des ouvrières, retirent des larves une partie importante de leur alimentation. Dans la plupart des sociétés pérennes des zones subdésertiques, le couvain est consommé de façon régulière au cours de la saison sèche, contribuant ainsi à la survie du groupe. Enfin, lors de la fondation d'une société (notamment lorsqu'elle est claustrale comme chez beaucoup de Fourmis supérieures) les œufs sont fréquemment dévorés soit par les larves, soit par la reine elle-même.

\section{II. - Relations parentales et évolution sociale.}

Registre fondamental des activités du grcupe, les relations parentales constituen également un bon « marqueur » du niveau d'évolution sociale. Leur étude, chez une espèce donnée, permet d'avoir une opinion approximative de la situation de cette dernière dans l'échelle évolutive. A titre d'exemples, chez les Abeilles primitives du groupe des Halictinés comme chez les Fourmis Ponérines, les femelles reproductrices peuvent participer activement aux soins à la progéniture au sein d'une société adulte. En revanche, dans les groupes plus spécialisés ce travail social n'est effectué par la femelle reproductrice que lors de la fondation de la société, c'est-à-dire dans la période se situant entre le « vol nuptial » ef l'apparition des premières ouvrières (à l'exception toutefois des espèces où la formation de nouvelles colonies se réalise par sociotomie, comme chez l'Abeille domestique ou des Fourmis Dorylines). Cette période de la fondation est souvent interprétée, probablement à juste titre, comme un atavisme nécessaire, reconstituant momentanément un maillon évolutif entre la vie solitaire et le début de la vie scciale, la femelle survivant à sa ponte et assurant la protection et le nourrissage des jeunes (n'oublions pas que chaque colonie d'Hyménoptère social est en fait un groupe familial). Chez les Guêpes et les Bourdons la femelle fondatrice réunit même les diverses potentialités des ouvrières puisqu'elle sort régulièrement du nid afin d'assurer l'approvisionnement. Parmi les Fourmis, le cas est courant dans les sous-Familles primitives mais absent dans les évoluées (fondation claustrale), à quelques très rares exceptions près, comme Neomyrma rubida, étudiée par Le Masne et Bonavita (1969), où la jeune reine sort chasser des proies afin d'en nourrir ses larves. Mais dans les groupes supérieurs ces activités de la femelle reproductrice cessent dès l'apparition des premières ouvrières. 


\section{III. - Les signaux impliqués dans les relations parentales.}

Presque toutes les analyses expérimentales des signaux impliqués dans les relations parentales chez les Hyménoptères sociaux sont particulièrement récentes : elles ont en général moins de dix ans. Les tests biologiques utilisés pour ces études tiennent compte des particularités éthologiques de chaque groupe.

Chez les Fourmis, on a généralement fait appel au transport du couvain par les ouvrières ef à son attractivité à l'égard de ces dernières. Watkins et Cole (1966) puis Schneirla (1971) ont décrit chez les Fourmis nomades (Dorylines) l'attraction des ouvrières pour du papier filtre imprégné d'odeur de couvain. Chez formica polyctena, la reconnaissance des nymphes par l'ouvrière adulte est réalisée grâce à une phéromone qu'elles portent au niveau cuticulaire (Jaisson, 1973). II s'agit d'une chimioréception de contact ou à très faible distance car la nymphe est perçue à travers son cocon de soie dont le réseau est suffisamment lâche pour favoriser la diffusion d'une substance ou même permettre la pénétration de l'extrémité de certains sensilles gustatifs de l'ouvrière. La substance (ou le groupe de substances) active semble être soluble dans l'eau ; elle est très stable et peu volatile, des nymphes préalablement tuées par congélation pouvant rester soignées encore pendant plusieurs dizaines de jours. Sa valeur est spécifique de l'espèce : des cocons hétérospécifiques, y compris du même genre, sont dévorés par l'ouvrière adulfe, tandis que ceux d'une autre colonie homospécifique sont adoptés sans difficultés. Walsh et Tschinkel (1974), critiquant les résultats antérieurs de Glancey et al. (1970), sont parvenus à un résultat voisin chez la « Fourmi de feu » américaine (Solenopsis invicta), en utilisant également des tests de transport. Chez cette Myrmicine la nymphe reste nue (aucun cocon n'esł tissé) et la substance active portée par la cuticule est reconnue des ouvrières directement par contact. Elle est soluble dans l'hexane mais perd son effel dans les 72 h qui suivent la mort de la nymphe. Pour cette même espèce, Bigley et Vinson (1975) ont isolé en outre une phéromone caractéristique du couvain de sexué, la trinoléine : des disques de papier filtre imbibés de cette substance ef déposés à l'extérieur sont saisis entre les mandibules et introduits au sein de la colonie où ils sont placés parmi le couvain de sexué, systématiquement séparé de celui d'ouvrière. Brian (1975) esł parvenu à la même conclusion chez Myrmica rubra, où les larves de sexués émettent un signal chimique particulier par leur surface ventrale. Ce signal s'ajouterait à un autre qui est général au couvain, constitué par une substance soluble dans l'acétone, l'éther et le chloroforme, très stable et non strictement spécifique de l'espèce (elle est également active sur Myrmica scabrinodis).

En ce qui concerne Guêpes et Apidés sociaux, c'est la thermorégulation qui fut plutôt utilisée comme test biologique dans l'analyse des signaux impliqués dans la reconnaissance du couvain. Chez la Guêpe Dolichovespula media, Ishay (1972) semble avoir démontré l'existence d'une phéromone produite par le couvain : des bandes de papier imprégnées d'extraits de nymphes déclenchent le processus de thermorégulation dans un nid où il avait été préalablement stopé par le retrait du couvain. Cependant, Koeniger (1975) obtient le maintien de l'activité thermorégulatrice chez le Frelon (Vespa crabro) en substituant aux nymphes de petites pierres; mais ce même résultat est impossible lorsque le nid est auparavant conservé vide pendant un an à 
une température de $20^{\circ} \mathrm{C}$. Toutefois, une réactivation du nid apparaît en lui ajoutant des nymphes ou un extrait de nymphes peu de temps avant le test. Dans ce cas, les ouvrières répondent donc à des signaux chimiques et mécaniques. En ce qui concerne l'Abeille domestique, Koeniger (1975) aboutit au même résultat d'un double signal mais la phéromone impliquée semble beaucoup plus labile que pour le Frelon. Enfin Heinrich (1974), utilisant des femelles fondatrices de deux espèces de Bourdons (Bombus vosnesenskii et edwardsii), a montré que le «comportement d'incubation », qui permet la régulation thermique, est induit par une phéromone spécifique de l'espèce non pas issue du couvain mais produite cette fois-ci par l'adulte lui-même, qui en imprègne le pollen qu'il met à la disposition des larves. Ainsi, cette fondatrice continuera à incuber pendant dix jours au moins une urne de cire d'où l'on aura préalablement retiré le couvain tandis qu'elle cessera immédiatement si ce dernier lui est restitué dans une urne remplie de pollen récolté par une espèce différente.

A côté de stimulations chimiques ef mécaniques peuvent aussi intervenir des stimulations acoustiques. Ishay et Landau (1972) l'ont montré chez le Frelon (Vespa orientalis) : les larves affamées produisent, en frottant les mandibules contre les parois de l'alvéole, des vibrations dont la propriété est d'exciter chez l'adulte le comportemenł de nourrissage.

\section{IV. - Déterminisme éthologique des relations parentales.}

Les caractéristiques du couvain, donc ses exigences de soin, évoluent : non seulement des différences évidentes existent entre œufs, larves et nymphes, mais on pense depuis longtemps (cf. par exemple Berlepsch, 1860, pour l'Abeille) que les signaux émis par les larves se modifient en fonction de leur âge, suscitant ainsi une évolution correspondante des soins. De même nous pensons que la nymphe de Fourmi proche de l'éclosion imaginale produirait un signal attractif (probablement chimique) déclenchant le léchage actif par les adultes. Cela aiderait la jeune ouvrière à se dégager de son exuvie nymphale mais aussi à capter l'odeur de la colonie, portée par ses sœurs aînées.

L'éthogenèse de l'imago a aussi ses conséquences sur les relations parentales. Cependant, peu de travaux se rapportent à cette question. Chez l'Abeille domestique, Rothenbuhler (1964) a démontré que le comportement «hygiénique», consistant à désoperculer les alvéoles contenant du couvain infecté par une bactérie pour le rejeter à l'extérieur de la ruche, est déterminé génétiquement par deux caractères récessifs non liés. Le premier commande la désoperculation de l'alvéole et le second le rejet. Cette réaction prophylactique s'avère très importante car elle permet de préserver le couvain sain. Ainsi, dans les régions touchées par la maladie, les races d'Abeille portant ces caractères résistent tandis que les autres sont décimées. Au contraire, en ce qui concerne la Fourmi rousse des bois (Formica polyctena) la reconnaissance des nymphes en vue de leur transport (voir Ch. III) n'est pas déterminée génétiquement mais dépend d'un apprentissage précoce (Jaisson, 1975). A l'éclosion, la jeune ouvrière n'est sensible qu'à un stimulus non spécifique de l'espèce : elle manifeste alors une attraction pour des cocons appartenant même à un genre différent (Camponotus ou Lasius). Il semble bien que la première semaine de vie imaginale 
constitue une période sensible, au terme de laquelle la jeune Fourmi ne manifeste plus de soins qu'au seul type de cocon connu, homo- ou hétérospécifique (Jaisson et Fresneau, 1978b). Quant aux groupes d'ouvrières isolés sans cocons depuis l'éclosion, ils deviennent inefficaces dans les activités de soins. Le Moli et Passetti (1977-78) ont retrouvé le même phénomène chez Formica rufa. II nous paraît très probable que la jeune ouvrière est d'abord sensible, sans apprentissage préalable, à une phéromone interspécifique (ce qui explique pourquoi elle répond positivement à des cocons de genres voisins). Il ne s'agit pas d'une substance unique pour toutes les Fourmis, mais au moins commune à plusieurs genres phylogénétiquement proches. En effet, aucune réponse adaptée n'apparait lorsqu'on propose à des très jeunes Fourmis les nymphes de certaines Myrmicines, sous-Famille différente (Jaisson, non publié). La substance inferspécifique serait associée, d'une façon qui reste à démontrer, à une seconde phéromene qui, elle, caractériserait l'espèce. Dans cette hypothèse, l'apprentissage précoce, voisin de ce que l'on appelle «l'empreinte », assurerait le relai entre le niveau spontané de sensibilité interspécifique et le niveau appris de sensibilité spécifique de l'espèce.

L'étude de l'éthogenèse chez les Hyménoptères sociaux, trop peu développée, nous semble fondamentale pour une meilleure compréhension des relations parentales. Les travaux récents de Topoff et Mirenda (1978) et Topoff et al. (1979) sur des Fourmis Dorylines d'Amérique tropicale (genre Neivamyrmex) vont d'ailleurs dans le même sens : le comportement particulier des jeunes ouvrières agit fondamentalement sur le cycle phase sédentaire-phase nomadique de la société.

La richesse ef la diversité des relations parentales chez les Hyménoptères sociaux s'expliquent par les différentes voies évolutives qui ont permis leur apparition mais aussi par le nombre très important d'espèces (près de 15000 probablement, rien que pour les Fourmis). Les caractéristiques propres à ces animaux, soulignées en introduction, la fonction sociale souvent très importante remplie par la progéniture, rendent qualitativement différentes les relations parentales par rapport aux Insectes solitaires ou aux Vertébrés. Ainsi par exemple, parmi les canaux sensoriels impliqués, la chimioréception se trouve considérablement privilégiée et diversifiée. Néanmoins, certains aspects se prêtent de plus en plus aisément à des comparaisons ou des synthèses avec les autres groupes : c'est le cas du déterminisme physiologique et du développement comportemental, qui suscitent d'ailleurs davantage l'intérêt des chercheurs. Il est donc probable qu'après une période où les différences se sont plutôt trouvées soulignées, nous arrivons à un moment où les essais de synthèse seront de plus en plus fréquents. Le présent colloque en est une illustration particulièrement significative.

Colloque «La relation parentale » Nouzilly, France, juin 1979.

\section{References}

BERLEPSCH A., 1860. Die Biene und ihre Zucht mit beweglichen Waben in Gegenden ohne Spätsommertracht. Ernst éd., Leipzig.

BIGLEY W. S., VINSON S. B., 1975. Characterization of a brood pheromone isolated from the sexual brood of the imported fire ant., Solenopsis invicta. Ann. Entomol. Soc. Amer., 68, 301-304.

BRIAN M. V., 1975. Larval recognition by workers of the Ant Myrmica. Anim. Behav., 23, $745-756$. 
FRESNEAU D., DUPUY P., 1980. La division du travail chez une Fourmi primitive du genre Neoponera par l'utilisation de méthodes statistiques modernes (à paraître).

GLANCEY B. M., STRINGER C. E., CRAIG C. H., BISHOP P. M., MARTIN B. B., 1970. Pheromone may induce brood tending in the fire ant, Solenopsis saevissima. Nature, 226, 863-864.

HAMILTON W. D., 1964. The genetical evolution of social behavior, I and II. J. theor. Biol., 7, 1-52.

HEINRICH B., 1974. Pheromone induced brooding behavior in Bombus vosnesenskii and B. edwardsii (Hymenoptera : Bombidae). J. Kans. Entomol. Soc., 47, 396-404.

ISHAY J., 1972. Thermoregulatory pheromones in wasps. Experientia, 28, 1185-1187.

ISHAY J., LANDAU E. M., 1972. Vespa larva send out rhythmic hunger signals. Nature, 237, $286-287$.

JAISSON P., 1973. L'imprégnation dans l'ontogenèse du comportement de soins aux cocons chez les Formicines. Proc. 7th. Congr. IUSSI, London, 176-181.

JAISSON P., 1975. L'imprégnation dans l'ontogenèse des comportements de soins aux cocons chez la jeune Fourmi rousse (Formica polyctena Först.). Behaviour, 52, 1-37.

JAISSON P., FRESNEAU D., 1978a. Evolution sociale chez deux Fourmis mexicaines. Film $16 \mathrm{~mm}, \mathrm{c}$. Prod. S.F.R.S., Paris.

JAISSON P., FRESNEAU D., 1978b. The sensivity and responsiveness of Ants to their cocoons in relation to age and methods of measurement. Anim. Behov., 26, 1064-1071.

KOENIGER N., 1975. Experimentelle Untersuchungen über das Warmen der Brut bei Vespa crabro und Apis mellifica. Verh. disch. zool. Ges., p. 148.

LE MASNE G., 1953. Observations sur les relations entre le couvain ef les adultes chez les Fourmis. Ann. Sci. nat., 15, 1-56.

LE MASNE G., BONAVITA A., 1969. La fondation des sociétés selon un type archaïque chez une Fourmi appartenant à une sous-famille évoluée. C. R. Acad. Sci. Paris, 269, 2373-2376.

LE MOLI F., PASSETTI M., 1977. The effect of early learning on recognition, acceptance and care of cocoons in the Ant Formica rufa L. Att. Soc. ital. Sci. nat. Mus. Civ. Nat., Milano, 118, $49-64$.

LE MOLI F., PASSETTI M., 1978. Olfactory learning phenomena and the cocoon nursing behaviour in the Ant Formica rufa L. Boll. Zool., 45, (sous presse).

LENOIR A., 1977. Sur un nouveau test éthologique permettant d'étudier la division du travail chez la Fourmi Lasius niger L. C. R. Acad. Sci. Paris, 284, 2557-2559.

MONTAGNER H., 1967. Comportements trophallactiques chez les Guêpes sociales. Film $16 \mathrm{~mm}$, c. Prod. S.F.R.S., Paris, No B-2053.

ROSCH G. A., 1930. Untersuchungen über die Arbeitsteilung im Bienenstaat. 2 Teil. Die Tätigkeiten der Arbeitsbienen unter experimentell veränderten Bedingungen. Zeit. Vergl. Physiol., 12, 1-71.

ROTHENBUHLER W. C., 1964. Behavior genefics of nest cleaning in honey-bees. IV. Responses of F1 and backcross generations to disease-killed brood. Amer. Zool., 4, 111-123.

SCHNEIRLA T. C., 1971. Army Ants : A sfudy in social organization. TOPOFF H. T. ed., Freeman, San Francisco.

TOPOFF H., MIRENDA J., 1978. Precocial behaviour of callow workers of the army ant Neivamyrmex nigrescens : importance of stimulation by adults during mass recruitment. Anim. Behov., 26, 698-706.

TOPOFF H., MIRENDA J., DROUAL R., HERRICK S., 1979. Effect of brood substitution in army ants (à paraître).

WALSH J. P., TSCHINKEL W. R., 1974. Brood recognition by contact pheromone in the red imported fire ant, Solenopsis invicta. Anim. Behav., 22, 695-704.

WATKINS J. F., COLE T. W., 1966. The attraction of army ant workers to secretion of their queens. Tex. J. Sci., 18, 254-265.

WILSON E. O., 1975. Sociobiology, The new synthesis. Belknap Press, Harvard, Massachusetts, 697 pp. 